\title{
New Developments on Calculating and Measuring \\ Ac Losses in Composite Superconductors
}

\author{
L.J.M. van de Klundert \\ University of Twente \\ Applied Superconductivity Centre \\ P.O. Box 217, 7500 AE Enschede, The Netherlands
}

\begin{abstract}
A method for the, complete numerical solution of the Maxwell equation in a multifilamentary wire carrying and a transport current and subject to applied fields of any direction compared with the axis of the wire, both current and applied fields of arbitrary time dependence has been developed. First results show unexpected differences from analytical models which were used in the past and are reviewed in this paper. From both approaches current degradation effects can be estimated and loss contributions resulting from combined action of applied field and current can be calculated.
\end{abstract}

\section{INTRODUCTION}

The present confidence in the rellability of composite superconductors has led to the design and construction of large and complex magnet systems. Accelerators, like the Tevatron and HERA rings do operate or will soon be in operation. The tokamak TORE SUPRA is in operation for some years. $T-15$ is in a test phase. Still larger systems are in a design phase and prototype components are constructed and subject to testing. Superconducting Magnetic Energy Storage, SMES, is reconsidered as a useful system for levelling off the imbalance between daily energy production and energy consumption patterns.

In all these, and other not-mentioned magnet. systems the electrodynamic behaviour of the strands used in the composite cables is basic to the final performance. In this paper we will reconsider the demands for these strands and outline some general aspects of their electromagnetic response. A proper model, suitable for solving the electromagnetic

† The author has deceased prior to publication of this paper.

Manuscript received June 24, 1991. This work is partially supported under NET contract 355/88-12/FU-NL. response consists of the following elements:

a. The position and time dependence of the applied field

b. Constitutive equations describing the local $J-E$ relations

c. The conductor layout.

As a results the following answers should be obtainable:

a. The low-loss current carrying capacity

b. The local and overall heat production.

Calculations of this type normally are performed under isothermal conditions. In principle, however; also the temperature distribution inside the conductor, possibly ending in a catastrophic temperature increase or quench, have to be evaluated. But this will not be the subject of this paper.

The current pattern inside a composite can be decomposed in three basic components each of which can be time dependent.

a. The transport current $I_{T}=\int_{A} j_{z} d A$.

b. Induced currents, which have closed loops inside one specific filament referred to as magnetization currents.

c. Induced currents which have closed loops consisting of superconducting and normal or matrix material. These currents are referred to as coupling currents. Under very special conditions also closed current loops entirely in a normal conducting region may be induced. But we will also consider these as coupling currents.

A break down of the heat production or total loss in transport current loss, magnetization loss and coupling current loss is only useful if no interaction between the various currents would exist which is normally not the case. Measurements to estimate magnetization and coupling loss contributions are therefore usually performed in absence of a transport current. The term eddy current loss is avolded because in the framework outlined above there is no need for it and it may be confusing since it is not defined there.

0018-9464/92\$03.00 ๑ 1992 IEEE 
II. THE MODEL

Throughout the rest of this paper we will use three coordinate systems simultaneously for clarity. If we follow the path of one strand in a fully transposed cable the local applied field components will vary periodically since the direction of the strand will change compared with the general direction of the applied fleld. Moreover, if a multi coil system like a tokamak is considered, also other period lengths of the applied field have to be considered. In Cartesian coordinates we therefore can write,

$$
B^{A}=\left(B_{x}^{A}, B_{y}^{A}, B_{z}^{A}\right),
$$

with the $z$-direction along the axis of the strand. Each of the components of $\mathbf{B}^{A}$ are a periodic function of $z$. Since it is convenlent to solve the Maxwell equations in cylindrical coordinates, they are also introduced in the usual way.

$$
\begin{aligned}
B_{r}^{A}= & B_{1} \sin (\varphi)+B_{2} I_{1}(p r) \sin (p z)+ \\
& B_{3}\left[I_{0}(p r)-I_{1}(p r) / p r\right] \sin (\varphi) \sin (p z) \\
B_{\varphi}^{A}= & B_{1} \cos (\varphi)+ \\
& B_{3} \frac{I_{1}(p r)}{p r} \cos (\varphi) \sin (p z) \\
B_{z}^{A}= & B_{z}^{0}+B_{2} I_{0}(p r) \cos (p z)+ \\
& B_{3} I_{1}(p r) \sin (\varphi) \cos (p z)
\end{aligned}
$$

Here $B_{\varphi}^{0}, B_{z}^{0}, B_{1}, B_{2}$ and $B_{3}$ are functions of $t$ only. $I_{0}$ and $I_{1}$ are Bessel functions of the second $k 1$ nd. Two cases will be considered, $B=\hat{B} \cdot e^{-i \omega t}$ the periodic time dependence and $B=0$ for $t<0$, and $B=\alpha t$ for $t \geq 0$ to study the transient phenomena.

The five coefficlents defined above refer to the following cases:

$B_{2}^{0}$ defines a uniform applied fleld parallel to the wire.

$B_{1}$ defines a uniform applied fleld perpendicular to the wire $\left(B_{y}^{A}=B_{1}\right)$

$B_{2}$ defines a perlodic applied fleld parallel to the wire with period length $L$ and $p=2 \pi / L$

$B_{3}$ defines a periodic applied field perpendicular to the wire again with period length $L$.

These five coefficlents can be chosen independently. Note that the components of $B^{\wedge}$ are chosen to satisfy $\nabla \cdot B^{A}=\nabla \times B^{A}=0$.
A third coordinate system is necessary to describe the constitutive equations. If we consider a local system with one component in the $r$-direction of the wire, one component parallel to the filament direction, and the third component perpendicular to the previous ones, we can write the constitutive equations

$\mathbf{j}=\left(\sigma_{\mathbf{r}} E_{\mathbf{r}}, \sigma_{\perp} E_{\mathcal{L}}, \gamma j_{c}+\sigma_{\|} E_{\|}\right)$.

The transformation of $\mathbf{j}$ from this local system to cylindrical coordinates is given by

$$
\begin{aligned}
& j_{r}=\sigma_{r} E_{r} \\
& j_{\varphi}=j_{\|} \sin \psi+\sigma_{\varphi \varphi} E_{\varphi}+\sigma_{\varphi z} E_{z} \\
& j_{z}=j_{\|} \cos \psi+\sigma_{\varphi z} E_{\varphi}+\sigma_{z z} E_{z} .
\end{aligned}
$$

Here: $\operatorname{tg} \psi=2 \pi r / L_{p}=\beta r, L_{p}$ is the twist length,

$$
\begin{aligned}
& \sigma_{\varphi \varphi}=\sigma_{\perp} \cos ^{2} \psi+\sigma_{\|} \sin ^{2} \psi \\
& \sigma_{\varphi z}=\left(\sigma_{\|}-\sigma_{\perp}\right) \sin \psi \cos \psi \\
& \sigma_{\mathrm{zz}}=\sigma_{\perp} \sin ^{2} \psi+\sigma_{\|} \cos ^{2} \psi .
\end{aligned}
$$

In this expression $\sigma_{r}, \sigma_{1}$ and $\sigma_{\|}$are independent.

The most important component of $\vec{j}$ is $j_{\|}$.

$$
j_{\|}=\gamma j_{c}+\sigma_{\|} E_{\|} \text {. }
$$

We will assume $\gamma=\eta \operatorname{sign}\left(E_{\|}\right)$if $E_{\|} \neq 0$

$|\gamma|<\eta$ if $E_{\|}=0$, $\eta$ being the volume fraction of superconductor.

A region where $E_{\|}=0$ is called unsaturated, whereas if $E_{\|} \neq 0$, a region is called saturated. In unsaturated regions the expression for $J_{\varphi}$ and $J_{2}$ reduce to

$$
\begin{aligned}
& J_{\varphi}=\beta r j_{s}+\sigma_{\perp} E_{\varphi} \\
& J_{z}=j_{s}+\sigma_{\perp} E_{z}
\end{aligned}
$$

with $J_{g}=\gamma j_{c} \cos \psi$, the superconducting component of $j_{z}$. It may be noted that if $E_{\|}=0, E_{z}=-\beta r E_{\varphi}$. This simplified formulation of the constitutive equation, where $\gamma$ is a non-linear function of $E_{\|}$only, is known as the anisotropic continuum model of Carr [1]. More complex expressions for $\gamma$ containing, apart from $E_{\|}$, the fllament radius $R_{f}$ and the local perpendicular fleld rate $\dot{B}_{\perp}$ were described by Rem [2] and Hartmann [3]. No attempts have been made yet to include $\dot{B}_{\|}$and $\left(E_{\perp}^{2}+E_{\Gamma}^{2}\right)^{1 / 2}$, the total electric fleld perpendicular to the filament. Also flux creep effects are not considered.

\section{LINEARIZATION OF THE PROBLEM}

The non-linear character of the problem resulting from the non-linear $j_{\|}$-component of the constitutive equation can be removed by assuming that the whole interior of the wire is unsaturated. In order to account for the boundary condition $E_{r}(R)=0$ or 
$B_{\varphi}(R)=B_{\varphi}^{0}$ in rotational symmetric problems a surface current $J$, which also behaves Iinearly, is introduced. $J$ flows in the filament direction and has components $J_{z}$ and $J_{\varphi}$, with $J_{\varphi}=\beta R J_{z}$.

If $E_{r}\left(R^{-}\right) \neq 0$ the condition $E_{r}\left(R^{+}\right)=0$ is fulfilled if

$$
\partial_{z} J_{z}+R^{-1} \partial_{\varphi} J_{\varphi}=\sigma_{r} E_{r}\left(R^{-}\right)
$$

$E_{r}\left(R^{-}\right)$and $E_{r}\left(R^{+}\right)$denote the limiting values for $r \rightarrow R$, if $r<R$ or $r>R$ respectively.

\section{A. Parallel field}

The shape of the applied field in Eq. 1 is chosen to be rotational symmetric. Then the Maxwell equations read

$$
\begin{aligned}
& -\partial_{z} E_{\varphi}=-\partial_{t} B_{r} \\
& \partial_{r} E_{r}-\partial_{r} E_{z}=-\partial_{t} B_{\varphi} \\
& \partial_{r}\left(r E_{\varphi}\right)=-r \partial_{t} B_{z} \\
& -\partial_{z} B_{\varphi}=\mu_{0} \sigma_{r} E_{r} \\
& \partial_{z} B_{r}-\partial_{r} B_{z}=\beta r \mu_{0} j_{s}+\mu_{0} \sigma_{\perp} E_{\varphi} \\
& \partial_{r}\left(r B_{\varphi}\right)=r\left(\mu_{0} J_{s}+\mu_{0} \sigma_{\perp} E_{z}\right) .
\end{aligned}
$$

If we, furthermore, assume that $L^{-1}=0$, .1.e. the problem is also z-invariant, we immediately get $E_{r}=B_{r}=0$ irrespective of the time dependence of $B^{A}$. Moreover, it can be shown that $E_{z}+\beta r E_{\varphi}=0$ implies $B_{\varphi}+\beta r B_{z}=0$ [4]. $E_{r}=0$ implies that no current exchange in the r-direction occurs, irrespective the value of $\sigma_{r}[5,6]$.

Two cases can be considered with respect to the time dependence:

$$
\begin{aligned}
& B_{z}^{A}=0 \quad t<0, B_{z}^{A}=\alpha t \quad t \geq 0 \quad \text { or } \\
& B_{z}^{A}=B_{0} e^{-1 \omega t} .
\end{aligned}
$$

For $E_{\varphi}$ a partial differential equation can be obtained,

$$
\partial_{r} \frac{1}{r}\left(1+\beta^{2} r^{2}\right) \partial_{r} r E_{\varphi}=\left(1+\beta^{2} r^{2}\right) \mu_{0} \sigma_{\perp} \dot{E}_{\varphi}
$$

The solution of this equation involves an infinite set of $\boldsymbol{\tau}$-values, which are not yet determined if $\mathrm{B}_{\mathbf{z}}^{\mathbf{A}}=\alpha \mathrm{t}$ is assumed. In the stationary case, 1.e. $t \gg \tau_{\max }, \tau_{\max }$ is the largest $t$-value, the solution reads

$$
\begin{aligned}
& E_{\varphi}=-\alpha \ln \left(1+\beta^{2} r^{2}\right) / 2 \beta^{2} r \\
& B_{\varphi}=\beta r \alpha t /\left(1+\beta^{2} r^{2}\right)-f(r) \\
& B_{z}=\alpha t /\left(1+\beta^{2} r^{2}\right)-g(r) \\
& \mu_{0} J_{S}=2 \beta \alpha t /\left(1+\beta^{2} r^{2}\right)^{2}-h(r)
\end{aligned}
$$

with

$$
\begin{aligned}
& \mathrm{g}=\frac{\alpha \mu_{0} \sigma_{\perp}}{4 \beta^{2}}\left[\left(1+\beta^{2} \mathrm{R}^{2}\right) \ln \left(1+\beta^{2} \mathrm{R}^{2}\right)-\right. \\
& \left.\quad\left(1+\beta^{2} r^{2}\right) \ln \left(1+\beta^{2} r^{2}\right)+\beta^{2}\left(\mathrm{R}^{2}-r^{2}\right)\right] \\
& \mathrm{f}=\beta \mathrm{rg} \\
& \mathrm{h}=2 \beta \mathrm{g} /\left(1+\beta^{2} r^{2}\right) .
\end{aligned}
$$

From (2) it can be seen, that if $\sigma_{\perp}=0 \quad(\tau=0)$ saturation occurs first if $r=0$ and

$$
\mathrm{B}_{\mathrm{z}}^{\mathrm{A}}=\alpha \mathrm{t}=\mu_{0} \mathrm{~J}_{c} \mathrm{~L}_{\mathrm{p}} / 4 \pi
$$

Thus saturation in the inner region occurs after a change in $B_{z}^{A}$ independent of the rate of change of $B_{z}^{A}$.

In case a sinusoidal time dependence of $B_{z}^{A}$ is assumed $B_{z}^{A}=B_{0} \cdot e^{-1 \omega t}$ it is useful to put

$$
\begin{array}{ll}
E_{\varphi}=1 \omega g, & E_{z}=-i \omega \beta r g \\
B_{\varphi}=\beta \partial_{r}(r g), & B_{z}=\frac{1}{r} \partial_{r}(r g), \quad \mu_{0} j_{s}=k .
\end{array}
$$

Writing

$$
g=A \sum_{n=0} a_{n} u^{2 n+1}, k=A \sum_{n=0} b_{n} u^{2 n}, \text { with } u=\beta r
$$

the following recurrence relations for $a_{n}$ and $b_{n}$ exist:

$$
\begin{aligned}
& a_{-1}=b_{-1}=0 \quad a_{0}=1 \\
& 4(n+1)(n+2) a_{n+1}=-\left[4(n+1)^{2} a_{n}+1 \omega t\left(a_{n}+a_{n-1}\right)\right] \\
& b_{n}=\beta^{2}\left[(2 n+1)^{2} a_{n}+1 \omega t a_{n-1}\right] \\
& \tau=\mu_{0} \sigma_{\perp} / \beta^{2}
\end{aligned}
$$

The constant A follows from the boundary condition for $\mathrm{B}_{\varphi}^{\wedge}=0$

$$
A=B_{0} / \beta\left(1+\beta^{2} R^{2}\right) \sum(2 n+2) a_{n}(\beta R)^{2 n}
$$

The loss power per unit of length can be determined from the real part of the Poynting vector

$$
\mathrm{S}=\frac{\pi \mathrm{R}}{\mu_{0}} \mathrm{E} \times \mathrm{B}^{*} .
$$

If we write

$$
S=\frac{\pi R^{2}}{2 \mu_{0}} i \omega B_{0}^{2}\left(\mu^{\prime}+1 \mu^{\prime \prime}\right)
$$

the real and imaginary part of $\mu=\mu^{\prime}+\mu^{\prime \prime}$ can be plotted for $\omega \tau$ and $\beta R$ as a parameter. Fig. 1 shows these curves. For $\beta R=0,1$.e. no twist, the curve for a pure metallic bar is found whereas for $\beta R \rightarrow 0$, i.e. the fllaments degenerate in rings, the semicircle of a paramagnetic material is obtained. 


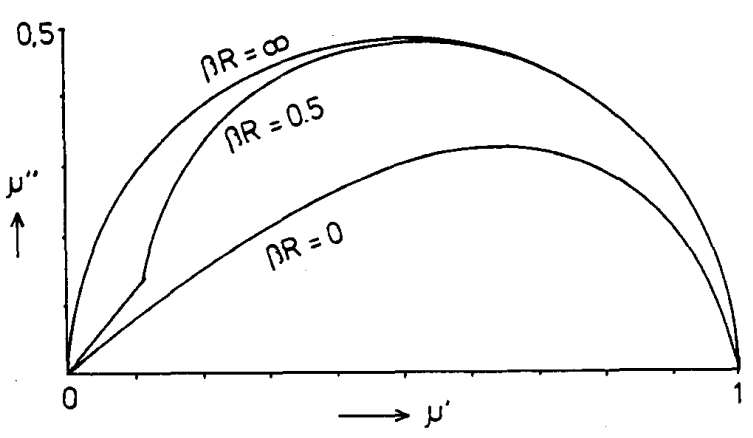

Fig. 1. Representation of the Poynting vector in terms of a complex permeability for a uniform parallel applied field.

\section{Numerical solutions}

In the previous subsection an analytical approximation for the maximum amplitude not saturating the interior of the wire and the loss power were given under the assumption that all return current flows in a surface shell. Numerical approaches to the problem show that some peculiar and unexpected effects are, however, present. Fig. 2 shows the time development of the saturated regions when a $z$-invariant $B_{z}^{A}=\sin (\omega t)$ is applied at $t=0$. When $\omega t<\pi / 2$ a negative saturated region starts in the outer region of the wire. At $\omega t=\pi / 2$ a positive saturated region starts at $r=R$ but the negative saturated region moves inward and

$\mathrm{t}(\mathrm{s})$

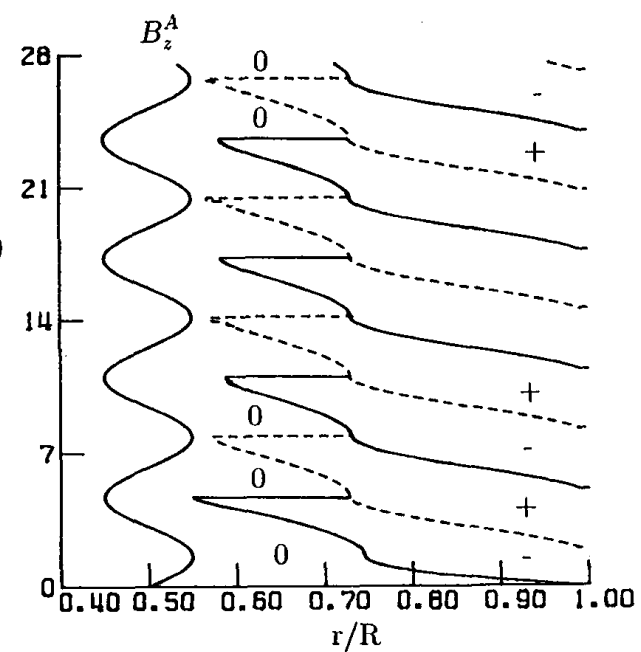

Fig. 2. Numerical solution of the saturation of the filaments for a uniform $B_{z}^{A}$ with sinusoidal time dependence and no transport current.

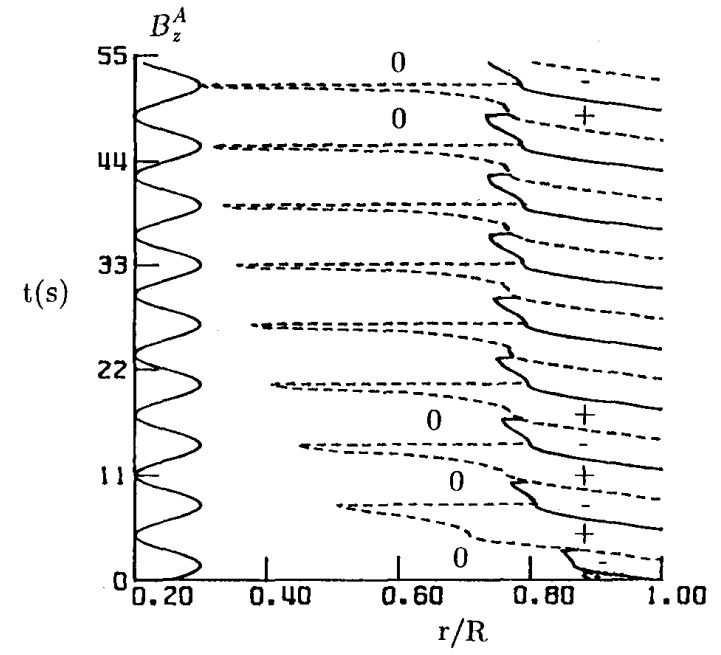

Fig. 3. Numerical solution of the saturation of the filaments for a uniform $B_{z}^{A}$ with sinusoidal time dependence with a transport current of about 0.2 of the critical current.

disappears at $\omega t=3 \pi / 2$. This pattern repeats each period. In this case no transport current is present. In Fig. 3 a small transport current is present and, as can be seen, the positive saturated regions penetrate much further than the negative ones. This means that a parallel applied field in an unexpected way reduces the current carrying capacity of the wire since at the outside a double layer of positive and negative saturated current exists. Numerical results for an applied field which is periodical in $z$ (Eq. 1) are shown schematically in Figs. 4 and 5. Fig. 4 shows the development of the saturated regions for increasing $B_{z}^{A}=\alpha t$. The upper picture shows the boundary of the outside region for small $t$. The middle picture shows the start of the development of the inner saturated region at $\mathrm{B}_{\mathbf{z}}^{\mathbf{A}} \sim \mu_{0} j_{\mathrm{c}} \mathrm{L}_{\mathrm{p}} / 4 \pi \quad$ [7] whereas the lower picture shows the final state of the saturated regions for $t \rightarrow \infty$. In Fig. 5 a schematic picture is given for sinusoidal applied non-uniform $B_{z}^{A}$ at some time. As in the uniform case in the outer region saturation in both directions coexists. The investigations are continued and more complete results will be published later.

\section{B. Perpendicular field}

In analogy with the previous treatment the case of a perpendicular applied field can be given. For reasons of brevity only the case of z-invariant sinusoidal time dependence of the applied field will be presented. For more detalls see $[8,9,10]$. 

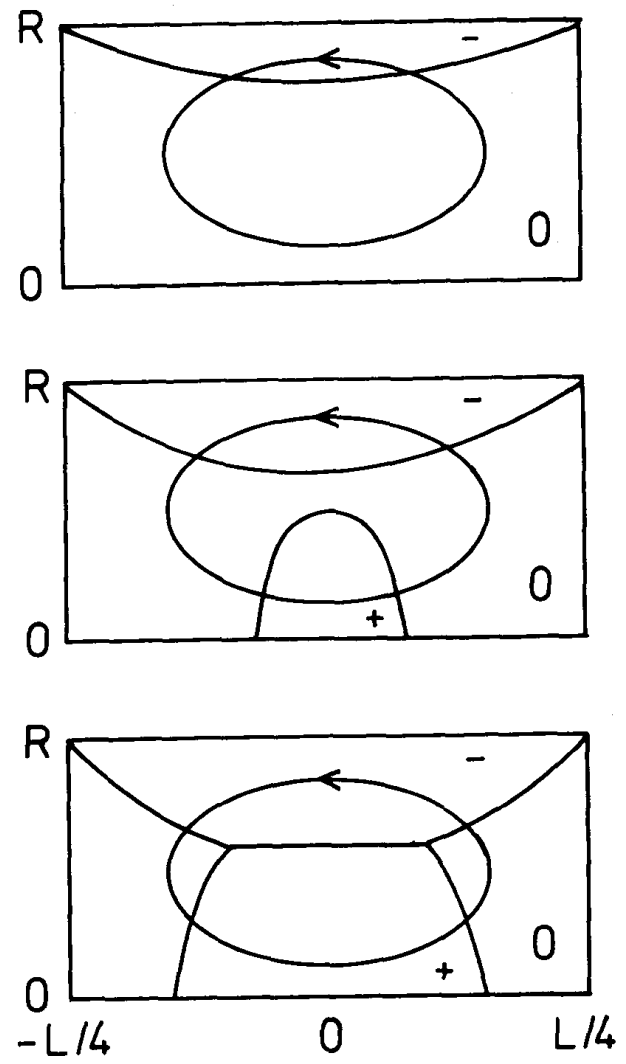

Fig. 4. The development of the saturated regions for periodic $B_{\mathbf{z}}^{A}$ and linear time dependence. For further explanation see text. The current pattern are indicated.

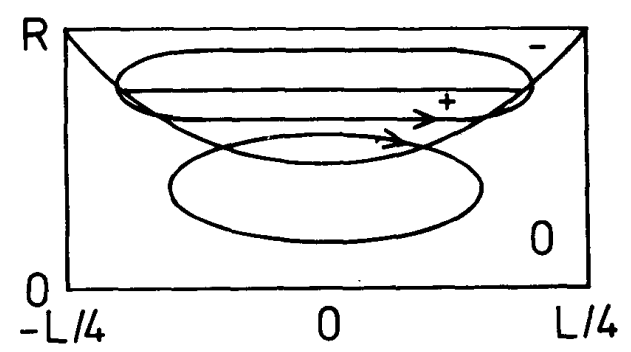

Fig. 5. Momentary solution of saturated regions for periodic $B_{z}^{A}$ with sinusoidal time dependence.

The Maxwell equations for this special case read

$$
\begin{aligned}
& \partial_{\varphi} E_{z}=i \omega r B_{r} \\
& -\partial_{r} E_{z}=i \omega B_{\varphi} \\
& \partial_{r} r E_{\varphi}-\partial_{\varphi} E_{r}=i \omega B_{z}
\end{aligned}
$$

$$
\begin{aligned}
& \partial_{\varphi} B_{z}=\mu_{0} \sigma_{r} r E_{r} \\
& -\partial_{r} B_{Z}=\beta r \mu_{0} j_{S}+\mu_{0} \sigma_{\perp} E_{\varphi} \\
& \partial_{r} r B_{\varphi}-\partial_{\varphi} B_{r}=r\left(\mu_{0} j_{S}+\mu_{0} \sigma_{\perp} E_{z}\right) .
\end{aligned}
$$

Assuming

$$
\begin{aligned}
& B_{r}=g \sin (\varphi) e^{-i \omega t} \\
& E_{r}=\frac{i \omega}{\beta} h \sin (\varphi) e^{-i \omega t} \\
& \mu_{0} J_{S}=i \omega \tau k \cos (\varphi) e^{-i \omega t},
\end{aligned}
$$

with $\tau=\mu_{0} \sigma_{\perp} / \beta^{2}$, we get for the surface current

$$
\mu_{0} \mathrm{~J}_{z}=-i \omega \tau R h(R) \cos (\varphi) e^{-i \omega t},
$$

and the remaining components of $E$ and $B$

$$
\begin{aligned}
& E_{\varphi}=\frac{i \omega}{\beta} g \cos (\varphi) e^{-i \omega t} \\
& B_{z}=-i \omega \tau \beta r h \cos (\varphi) e^{-i \omega t} \\
& B_{\varphi}=\partial_{\Gamma}(r g) \cos (\varphi) e^{-i \omega t} .
\end{aligned}
$$

Putting

$$
g=A \sum_{n=0} a_{n} u^{2 n}, \quad k=A \sum_{n=0} b_{n} u^{2 n}, \text { with } u=\beta r
$$

again recurrence relations for $a_{n}$ and $b_{n}$ can be derived.

$$
\begin{aligned}
& a_{-1}=b_{-1}=0 \quad a_{0}=b_{0}=1 \\
& 4 n(n+1)(1-i \omega \tau) \quad a_{n}=i \omega \tau\left[\left((2 n+1)^{2}-1+i \omega \tau\right) b_{n-1}\right. \\
& \left.\quad-(2 n+1) a_{n-1}\right] \\
& 4 n(n+1)(1-i \omega \tau) \quad b_{n}=i \omega \tau\left[i \omega \tau(2 n+1) b_{n-1}-a_{n-1}\right]
\end{aligned}
$$

From the boundary conditions A can be determined to be

$$
A=2 B_{0} /\left[g(\beta R)+\left(1-i \omega \tau\left(1+\beta^{2} R^{2}\right)\right) h(\beta R)\right] .
$$

and consequently the power loss per unit length

$$
\mathrm{S}=\frac{\pi \mathrm{R}}{2 \mu_{0}} \mathrm{E}_{\mathrm{z}} \mathrm{B}_{\varphi}^{*} .
$$

If we write again

$$
S=\frac{\pi R^{2}}{2 \mu_{0}} 1 \omega B_{0}^{2}\left(\mu^{\prime}+i \mu^{\prime \prime}\right)
$$

the values of $\mu^{\prime}$ and $\mu^{\prime \prime}$ can be plotted as shown in Fig. 6.

For small $\beta$ the $\mu^{\prime \prime}\left(\mu^{\prime}\right)$ curve has an ellipse as a limiting envelope whereas for $\beta \rightarrow \infty$, where the filaments degenerate in rings, the relation for a solid copper wire 


$$
\mu=1+J_{2}(k r) / J_{0}(k r)
$$

is obtained. ( $\left(k^{2}=1 \omega \mu_{0} \sigma_{\perp}, J_{0}\right.$ and $J_{2}$ Bessel functions). It may be noted that for small $\omega$ the power loss is

$$
P=\frac{\omega B_{0}^{2}}{\mu_{0}} \frac{\omega \tau^{\prime}}{1+\left(\omega \tau^{\prime}\right)^{2}} \text {, with } \tau^{\prime}=\frac{\mu_{0} \sigma}{2}\left[\left(\frac{L_{p}}{2 \pi}\right)^{2}+R^{2}\right]
$$

rather than the widely used expression for $\tau$ where the term $R^{2}$ is omitted.

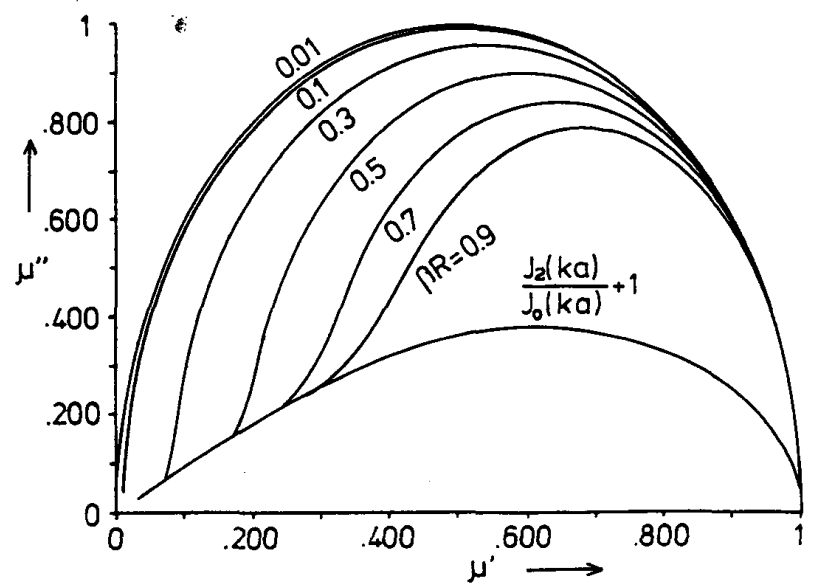

Fig. 6. Representation of the Poynting vector in terms of a complex permeability for a uniform perpendicular applied fleld.

\section{CONCLUSIONS}

Reliable calculations of the loss and current carrying capacity of composite wires in perlodic parallel and or perpendicular fleld can only be performed numerically. Apart from the time dependence also the spatial dependence along the wire should be considered. A Fourier expression seems to be sufficient. Analytical approaches using a surface current approach (1inearization) are not sufficient accurate for parallel fields of large amplitude because they do not correctly take into account the extension of the saturated regions. Further investigations are necessary.

\section{ACKONOLLEGEMIENTS}

The author is very much indebted to Prof. P.J. Zandbergen and F.P.H. van Beckum for their continuous interest and stimulating discussions in the problems of electrodynamics in composite superconductors and to E.M.J. Niessen and J.A. Eikelboom allowing me to use their results prior to publication.

\section{REFERENCES}

[1] W.J. Carr, jr., "Electromagnetic theory for filamentary superconductors", Phys. Rev., vol. B 11, 1975, pp. 1547-1554.

[2] P.C. Rem "Numerical models for AC superconductors", Ph.D. Thesis University of Twente, Enschede, The Netherlands, 1986.

[3] R.A. Hartmann, "A contribution to the understanding of AC losses in multifilamentary superconductors", Ph.D. Thesis University of Twente, Enschede, The Netherlands, 1989.

[4] E.M.J. Niessen, A. Nijhuis, E.J. Oetsen and L.J.M. van de Klundert, "Current degradation and coupling losses in ring shaped wires due to applied magnetic field changes", this conference.

[5] B. Turck, "Courants de circulation et pertes dans les composites supraconducteurs soumis a une induction longitudinale variable", Revue de Physique Appliquée, 1976, p. 369

[6] G. Ries and K.P. Jüngst, "Filament coupling in multifilamentary superconductors in pulsed longitudinal fields", Cryogenics, vol. 26, 1976.

[7] L.J.M. van de Klundert, G.B.J. Mulder, E.M.J. Niessen and A.J.M. Roovers, "Calculation and measurement of AC losses in NET OH and TF conductors", Final report NET contract 355/88-12/FU-NL, 1991.

[8] J.A. Eikelboom, "AC losses in prototype conductors for the NET toroidal field coils", Ph.D. Thesis University of Twente, Enschede, The Netherlands, Oct. 1991.

[9 ] L.J.M. van de Klundert, E.MJ. Niessen, P.J. Zandbergen, "Electrodynamics of composite superconductors", to be published.

[10] E.MJ. Niessen, LJ.M. van de Klundert, "Superconducting multifilamentary wires in inhomogeneous perpendicular a.c. fields", Proceedings of the international symposium on $A C$ superconductors, Smolenice, CSFR, 1991, ISBN 80-900506-0-3. 